\title{
The Day after Tomorrow
}

\author{
Erhard Taverna
}

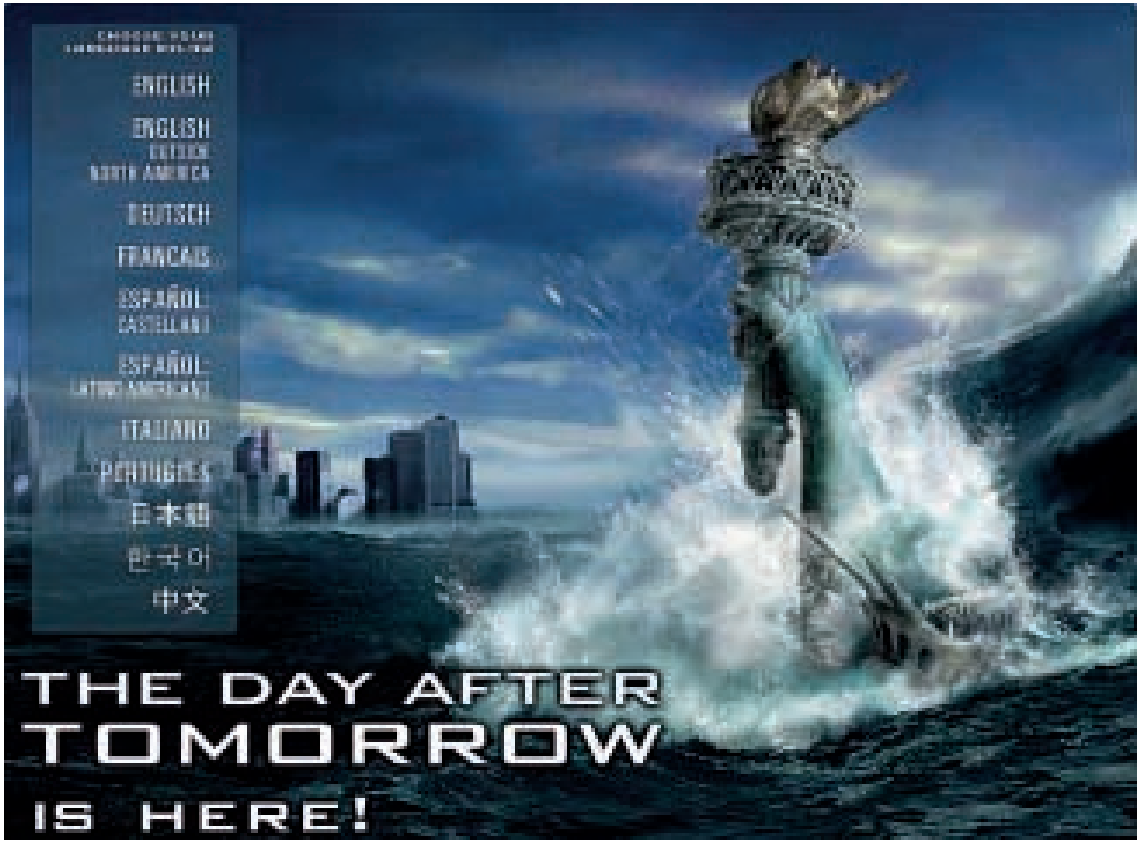

Die Erdbeben, die am 1. November 1755 Lissabon vollständig zerstörten, waren auch ein mediales Ereignis. Sie erschütterten erstmals die ganze europäische Öffentlichkeit. Neben dem traditionellen Verständnis im Sinne der Vergeltungstheologie, die in jeder Katastrophe eine Strafe Gottes wahrnahm, gab es auch Bemühungen für zahlreiche seismische Beobachtungen, eine rationale Erklärung zu finden. Grosse Geister wie Voltaire und Haller gerieten sich unversöhnlich in die Haare über die theologischphilosophische Frage, ob alles in dieser Welt gut sei.

Heute ist es denkbar und wahrscheinlich, dass menschliches Handeln allein für eine globale Katastrophe ausreicht. Szenarien zum «nuklearen Winter» werden durch neue Risiken wie den «Treibhauseffekt» abgelöst. Der schwäbische Regisseur Roland Emmerich verwandelt die nördliche Hemisphäre in eine Eiswüste. Ein gigantischer Katastrophen-Action-Thriller, der den Weltuntergang im Zeitraffer vorführt. Das ewige Eis der Antarktis zerbröselt minutenschnell und bringt mit seinen Kaltwassermassen die Warmwasserpumpe der Atlantikströme zum Stillstand. Ein Abbruchuniversum ist die Folge, mit grandiosen Bildern der Zerstörung, durch baseballgrosse Hagelkörner über Tokio, riesige
Tornados, die L.A. verwüsten, und monströse Flutwellen in Manhattans Häuserschluchten. Sintflutartige Regengüsse ersäufen die Kontinente, Europa erstickt unter einer fünf Meter hohen Schneelast, vom schottischen Himmel fallen Helikopter wie erfrorene Vögel. Schockgefroren imponiert die havarierte Skyline New Yorks, aus deren Eishölle die Freiheitsstatue unbeirrt die erloschene Fackel hochhält. Das gewaltigste Wettertief aller Zeiten entfaltet in seinen besten Teilen eine mitreissende visuelle Wucht. Der grossartigen, computergenerierten Umsetzung steht leider eine dürftige, klischeehafte Geschichte gegenüber: Ein prophetischer Klimatologe als heldenhafter Superpapi, der seinen genialen Sohn rettet, während das ebenso tapfere Mami als Ärztin bei einem krebskranken Kind ausharrt. Die einen überleben am idyllischen Kaminfeuer aus Bibliotheksbüchern im Tiefkühlinferno, Hunderttausende flüchten illegal über die mexikanische Grenze nach Süden. Am Ende dankt ein geläuterter Vizepräsident der dritten Welt für die gastliche Aufnahme, und die Crew von der Weltraumstation vermeldet eine neue, eiszeitliche Welt, überwölbt vom glasklar gesäuberten Himmel. Dem entvölkerten Norden ist nach dem stürmischen Purgatorium wenigstens ein Original der Gutenberg-Bibel erhalten geblieben.

«Am Tag danach» ist der Titel einer Buchausgabe «Zur Bewältigung von Naturkatastrophen in der Schweiz 1500-2000»; von der «Buzza di Biasca», einer Überschwemmung, die im Jahr der Schlacht von Marignano 1515 das Tal des Ticino verheerte, bis Gondo am 15. Oktober 2000. An Animationen und Tricks kann das Buch nicht mit dem Film mithalten. Dafür ist die Botschaft gründlich recherchiert und spannend dargestellt. Natur- und Sozialwissenschaften haben für einmal vorbildlich zusammengearbeitet für eine Darstellung, die wissenschaftlichpraktische und geschichtliche Perspektiven verbindet. Wie wurden diese Katastrophen wahrgenommen, wie reagierten Bund und Kantone, was hatten sie für Folgen? Das gut illustrierte Werk vereinigt intelligente Aufsätze aus verschiedenen Fachgebieten zu einer Gesamtschau, die aus der Analyse von Lernprozessen Strategien der Risikobewältigung erarbeitet. Ursprünglich als Thema für die Expo.02 konzipiert, haben die 19 Direktoren der kantonalen Gebäudeversiche- 
rungen und die Interkantonale Rückversicherung IRV das Folgeprojekt zusammen mit dem Schweizerischen Nationalfonds ermöglicht. Eine deutsche und eine französische Ausgabe wollen die landesweite Solidarität betonen.

Eingangs wird aus «Der Mensch erscheint im Holozän» von Max Frisch zitiert: «Katastrophen kennt allein der Mensch, sofern er sie überlebt. Die Natur kennt keine Katastrophen.»

Morgen oder übermorgen, die Ähnlichkeit der Titel ist zufällig und vielleicht doch programmatisch. Die Ökodebatte braucht sie beide: den weltweit vermarkteten Montagevirtuosen und Bildermacher wie Emmerich, gleich wie das leisere, auf die Lokalgeschichte beschränkte Autorenkollektiv. $\mathrm{Zu}$ ergänzen wären sie durch eine ebenso effektsichere Darstellung der schleichenden Katastrophen täglicher Umweltzerstö- rung, die wir in unserer Kurzlebigkeit übersehen. Naturkatastrophen wurden häufig als Vorzeichen des Jüngsten Gerichts gedeutet, als Wunderzeichen, die zur moralischen Umkehr aufriefen. Inzwischen wurde die Theodizee fallengelassen. Die Übel in der Welt sind nicht Gott anzulasten. Denn die Folgen menschlicher Dummheit wurden zum Forschungsobjekt. Leichter als die Theologie wird es die Wissenschaft nicht haben.

- Christian Pfister. Am Tag danach / Le jour d'après.

Bern: Paul Haupt Verlag; 2002. 263 Seiten.

- $\quad$ The Day after Tomorrow.

Verleih Fox, Laufzeit 123 Min.

Regie: Roland Emmerich,

Drehbuch: Nachmanoff, Emmerich.

\section{Kuschelkinder}

\section{B. Gurtner}

Korrespondenz:

Dr. med. Bernhard Gurtner

Eggstrasse 76

CH-8620 Wetzikon
Wenn die Mädchen zur Mandeloperation auf die Kinderabteilung kamen, brachten sie ihre liebste Puppe mit. Die nach einem Velounfall eingelieferten Buben baten ihr Mami, nachträglich den Teddybär zu bringen. Nachts schliefen die kleinen Patientinnen und Patienten mit ihrem Kuschelliebling im Arm, tagsüber musste mancher Teddy die gleichen Spitalplagen erleiden wie sein Besitzer. Die geduldige Krankenschwester verabreichte ihm eine Spritze mit Kochsalzlösung, um dem ängstlichen Kind zu zeigen, dass die Injektion gar nicht so weh tut. Auch die Puppen mussten ganz ruhig liegen und durften das Pflaster am Bauch oder den Kopfverband nicht wegreissen. Dauerte der Klinikaufenthalt länger, kamen weitere ausgestopfte Liebesgaben dazu, bis der sich ansammelnde Zoo von Schäfchen, Kätzchen, Hunden, Äffchen, schwarzweissen Pinguinen und grellgrünen Fröschen das Kinderbett zur Arche Noah werden liess. Das freute das Pflegepersonal genausowenig wie die unzähligen Blumen, die für erwachsene Kranke mitgebracht wurden.

Puppen und allerlei Plüschtiere werden auch heute noch mit ins Spital genommen, obwohl die elektronisch beseelten Pokémons und Tamagotchis zeitweise die traditionellen Lieblinge verdrängt hatten, die ja nur die Glasaugen rollen oder mit «Bääh» antworten konnten, wenn man ihnen auf den Bauch drückte. Die Pokémons heissen Rattfratz, Taubsi, Pikachu, Hornliu oder
Pummeluff und lassen sich im Computerspiel als pflegebedürftige Babys verwöhnen. Auch die vom Planeten Tamagotchi stammenden japanischen Cyberspace-Pets überleben umso länger, je aufmerksamer ihre Bedürfnisse durch wiederholten Tastendruck erfüllt werden. Sie brauchen regelmässig Sonnenschein, man muss sie füttern oder mit ihnen spielen, bis sie signalisieren, dass sie happy sind. Sogar ihr Hinterteil muss sauber gereinigt werden, sonst kehren sie beleidigt auf ihren fernen Stern zurück.

Die Pokémons und Tamagotchis sind schon wieder ausser Mode gekommen. Die weichen Plüsch- und Pelztierchen haben überlebt und werden in jedem Supermarkt, an Tankstellen und Kiosken angeboten. Man findet sie sogar in den Gemischtwarenhandlungen der Post, wo nun auch Schleckereien verkauft werden, seitdem die Briefmarken nicht mehr mit eigenem «Speuz» befeuchtet werden müssen. Auch erwachsene Kunden wollen im täglichen Stress ihr Lutschbedürfnis und Streicheldefizit befriedigen. Und so ist zu unserem Erstaunen schon vor längerer Zeit ein 30jähriger Prokurist mit seinem grossen Teddybär ins Spital eingerückt. Der Mann schien psychisch unauffällig und erwies sich nur als Vorbote eines Trends, der seither gang und gäbe geworden ist. Dem Teenageralter längst entwachsene Patientinnen bringen ihre Puppe mit, Besucher schenken den Kranken flauschige Kuscheltierchen oder finden 
es originell, einen grossen Stoffbernhardiner als Wachhund neben das Klinikbett zu stellen.

Jüngere Spitalangestellte dünkt das ganz normal, wir Älteren hatten damit etwas Mühe und fragten uns, ob die nachkommende Generation ein unerfülltes Liebesbedürfnis habe oder ob gerade umgekehrt uns selbst eine Zärtlichkeit fehle, die heute in aller Offenheit gezeigt und gelebt wird. Können warm anzufühlende Stofftiere das ersetzen, was an mitmenschlicher Zuwendung fehlt? Will man in die stummen Wesen alles hineinlegen, was einen bedrückt, ohne befürchten zu müssen, dass sie die Hand beissen, welche sie streichelt? Jedenfalls sind Plüschwesen weniger anspruchsvoll als ein lebendiger zwei- oder vierbeiniger Partner.

Im Alterspflegeheim, wo die betagten Bewohner oft auch wieder etwas kindlich oder gar kindisch werden, haben die Kuscheltiere schon lange einen festen Platz. Sie trösten darüber hinweg, dass Besucher ausbleiben und eigene Haustiere nur selten mitgenommen werden dürfen. Machte man eine Ausnahme und liess eine Katze $\mathrm{zu}$, wurde sie von den anderen Insassen so oft gefüttert, dass sie wegen Herzverfettung noch vor ihrer Besitzerin starb. Manchmal war es auch notwendig, einen schon räudig gewordenen uralten Teddybär gründlich $\mathrm{zu}$ waschen und $\mathrm{zu}$ desinfizieren, wobei sein versabbertes Fell noch die letzten verbliebenen Haare verlor. Der als Ersatz beschaffte goldgelbe neue Teddy fand keine Gegenliebe und wurde vom Beschenkten schnöde verstossen. Ebenso wenig erfreut zeigten sich in einem TV-Beitrag die schreienden, kurz zuvor durch Kaiserschnitt entbundenen Zwillinge, für die im vorgewärmten Säuglingsbett einer modernen Gebärklinik schon zwei feinste Plüschtierchen bereitlagen. Von der Wiege bis zur Bahre hält man sich an Kuschelware?

Psychologen und Soziologen haben dieses Phänomen einer seit 1968 nie mehr erwachsen werdenden Gesellschaft schon oft diskutiert und je nach Standpunkt gegenüber Plüsch und Plausch begrüsst oder beklagt. Gut sei es, wenn man das kindliche Staunen, Fragen und die jugendliche Entdeckerfreude ein Leben lang bewahre, kindisch hingegen, wenn man sich von Moden, Medien und Machtmenschen folgsam gängeln lasse. In der Bibel steht zwar: «Wenn ihr nicht werdet wie die Kinder ...», doch das Teddybärli-Schmusen der volljährigen Kuschelknaben war damit wohl nicht gemeint. Oder vielleicht doch ein ganz klein wenig?

PS. Wie der NZZ vom 28. Mai 2004 zu entnehmen war, will die Zürcher City-Vereinigung im Sommer 2005 die Limmatstadt mit Hunderten künstlerisch gestalteten Teddybären verschönern. Nach den zahmen Löwen, bemalten Kühen und schiefen Sitzbänken folgt ein sanfter «Teddy-Summer» für einheimische und zugereiste Kuschelkinder. 